\title{
L'abus de Daturas et de Kava en Nouvelle Calédonie : une pratique inquiétante
}

\section{Addiction involving Daturas and Kava in New Caledonia}

\section{Yann BARGUIL (1)*, Sylvain MERMOND ${ }^{(1)}$, Pascal KINTZ(2), Marion VILLAIN ${ }^{(2)}$, Erwan CHOBLET ${ }^{(1)}$, Vincent CIRIMELE ${ }^{(2)}$, Pierre CABALION ${ }^{(3)}$, Daniel DUHET $^{(3)}$, Jean-Yves CHARLOT ${ }^{(4)}$}

(1) Centre Hospitalier Territorial de Nouvelle-Calédonie, Laboratoire de Biochimie et d'Hémiostase, BP J 5 - 98849 Nouméa

(2) ChemTox- 67400 Strasbourg

(3) Institut de Recherche pour le Développement (4) Centre Hospitalier Spécialisé, Nouméa - Nouvelle-Calédonie

*Auteur à qui adresser la correspondance : Yann BARGUIL, Centre Hospitalier Territorial de Nouvelle-Calédonie, BP J 5 - 98849 Nouméa - Nouvelle-Calédonie

(Reçu le 20 janvier 2006 ; accepté le 13 février 2006)

\section{$R \hat{E} S U M E ́$}

En Nouvelle-Calédonie ( $N$-C), pays français du Pacifique Sud-Ouest, deux tendances inquiétantes émergent et ne doivent pas être ignorées: les consonmations de daturas, appelées Herbe du Diable, et celle abusive d'une boisson tranquillisante océanienne faite à partir du rhizome d'un poimier, le kava, surnomzné Narcotic Pepper par A. Shulgin. Nous décrivons pour chaque plante une série de cas cliniques et/ou médico-légaux récents.

Les daturas (Datura sp. \& Brugmensia sp.) sont des solanacées herbacées ou arborescentes. Six espèces ont été introduites en $\mathrm{N}$-C. Toutes les parties de la plante sont réputées contenir des alcaloüdes tropaniques dont l'atropine, mélange de $D$ et $L$-hyoscyamine et surtout, la scopolamine qui prédomine dans les daturas présents en N-C. Ces deux agents antimuscariniques sont responsables, selon la dose, de sédation ou de délire hallucinatoire - parfois cauchemardesque recherché par les usagers. Le diagnostic doit pouvoir écarter un premier épisode de psy'chose et l'analyse toxicologique prend toute son importance. Sur l'année 2005, nous rapportons les cas de 4 hommes âgés de 20 à 28 ans hospitalisés pour troubles sévères du comportenent après pour 3

\section{SUMMARY}

In New Caledonia $(N-C)$, French country of South Pacific, there are two new emerging problems : consumption of Daturas, so-called "Herbe du Diable" and abuse of a typical sedative drink obtained from the roots of a Pepper tree, "kava", that is known as "Narcotic Pepper", according to A. Shulgin.

For each plant, we describe a series of clinical and/or forensic cases.

Daturas (Datura sp. \& Brugmensia sp.) are belonging to the Solanaceas group. Six species have been introduced in N-C. All the parts of the plant contain tropanic alkaloids, such as atropine, a mixture of $D$ and L-hyoscyamine and mostly scopolamine. These drugs are involved in the hallucinogenic and sedative effects.

In 2005, we have observed 4 cases involving men, aged 20 to 28 years and hospitalized after severe impairment. HPLC/DAD was useful for the identification of scopolamine in the preparations, but did not reach the requested sensitivity for biological (blood and urine) specimens. For that, LC/MS-MS appears as the method of choice. Moreover; using this technique, segmental hair analysis demonstrated 
cas, l'ingestion de tisane du Diable et pour le $4^{\text {imte }}$, la fumigation dans un shilom de la plante associée à de la marijuana (dont les teneurs en THC variaient en 2005 de 0,25 à $13,8 \%)$. Les analyses toxicologiques ont été réalisées par HPLC/DAD et par LC/MS-MS. Alors que l'HPLC/DAD mettait en évidence la scopolamine dans les parties végétales (feuilles : 0,80 à 10,60 $\mu \mathrm{g} / \mathrm{m} g$, fleurs entières : 6,30 à 10,60 $\mu \mathrm{g} / \mathrm{mg}$, poids sec) et dans des décoctions de Brugmensia sp. (30 à $37 \mathrm{mg} / \mathrm{L}$ ), elle ne nous a jamais permis de retrouver les principes actifs dans le sang ou l'urine des sujets. Dans ces milieux, seule la LC/MS-MS a permis cette détection. Chez un sujet, la LC/MS-MS a prouvé par analyse capillaire séquentielle la consommation chronique de Datura inoxia. Par contre, l'analyse de poils de barbe d'un consommateur occasionnel s'avérait négative.

Piper methysticum, communément appelé kava est une pipéracée traditionnelle du Pacifique dont les racines sont importées en $N$-C. La racine est broyée, puis malaxée dans l'eau, la solution est flltrée et consommée dans des bars à kava ou nakamals. Les substances actives sont des lactones aux effets benzodiazépines-like. La consonnnation de cette boisson n'est pas réglenentée en $N$-C. Or; depuis quelques années, on relève des formes d'abus de kava à type d'ingestion massive ou de polyusage l'associant à l'alcool et au cannabis, cette demière défonce étant communément nommée " trithérapie » ou " triathlon » par les usagers euxmêmes. A titre d'exemple, nous rapportons 4 cas médicolégaux survenus durant le seul mois de septembre 2005 concernant 2 hommes impliqués dans 2 rixes dont l'une mortelle, et 2 femmes dont l'une était retrouvée morte à son. domicile. Leurs âges étaient compris entre 24 et 30 ans. Les analyses toxicologiques étaient réalisées sur le sang par HPLC/DAD. Chez les 2 hommes, le kava était associé au cannabis; chez la jeune fenme, des kavalactones ont été retrouvées en association à de la cyamémazine qui lui était prescrite.

De l'ethnomédecine aux nouvelles conduites addictives : sans références culturelles, l'usage des plantes psychotropes est dévoyé et ouvre la porte aux abus. Il s'agit surtout d'un polyusage associant plantes, alcool et cannabis à but avoué de défonce par potentialisation de leurs effets. Le prétexte d'un retour à la tradition ou d'une néo-tradition ne peut excuser ces abus qui doivent être officiellement dénoncés par les instances gouvernenentales. Le « secret-défonce 》doit être levé et une réglementation défunie.

\section{MOTS-CLÉS}

Datura, kava, tradition, Nouvelle Calédonie.

\section{Introduction}

La Nouvelle-Calédonie est un pays français du Pacifique Sud-Ouest où le cannabis connaît des conditions climatiques idéales à sa croissance. La côte Est (au vent) et les Iles Loyauté ainsi que l'Ile des Pins sont particulièrement propices à la production de marijuana à teneurs en THC élevées, approchant par endroits les $14 \%$ (1). A notre connaissance, la production est consommée localement ou vendue sur la capitale Nouméa - générant d'importants revenus pour les producteurs. Il n'y aurait pas d'exportation à l'heure in one subject a chronic abuse of Datura inoxia. However, in another situation, beard hair of a chronic abuser remained negative.

Piper methysticum, generally called "kava" is an original plant of the Pacific. The root is grinded, mixed with water and after filtration, the preparation is consumed in specific places, such as kava bars or nakamals. Active ingredients are lactones, with benzodiazepine-like effects. Consumption is not forbidden in $\mathrm{N}-\mathrm{C}$, and frequently associated with alcohol and carnabis. To document this specific abuse, we report 4 forensic cases observed during September 2005. Kavä̈ne, tested by $L C / D A D$, was associated with cannabis in two cases and with cyamemazine in one case.

These two typical forms of addiction appear as a new way of polydrug abuse, imolving traditional plants, cannabis and alcohol. Deep impairment is observed, due to potentialisation of the active compounds. Old traditions cannot support this behaviour and politics should take it into consideration to propose a suitable legislation.

\section{KEY-WORDS}

Datura, addiction, kava, New Caledonia.

actuelle. Cette culture se fait aux dépens de l'agriculture vivrière qui est délaissée au profit de cette production de drogue, générant une économie parallèle bien plus lucrative.

La consommation d'alcool est aussi importante. L'ivresse aigüe est responsable de violences diverses et on compte trois fois plus d'accidents mortels en Nouvelle-calédonie qu'en Métropole. Le Gouvernement local a pris la mesure de ces fléaux et « les addictions » sont un des thèmes de santé principaux retenus pour l'année 2006.

Cependant, deux tendances inquiétantes émergent et ne doivent pas être ignorées : la consommation de « Tisane du Diable » à base de daturas et celle, souvent abusive, d'une boisson tranquillisante océanienne préparée à partir du rhizome d'un poivrier, le « kava », surnommé « Narcotic Pepper » par Alexander Shulgin (2).

Afin d'illustrer notre propos, nous décrivons une série de cas cliniques et/ou médico-légaux récents pour chaque plante.

\section{Les daturas néo-calédoniens}

Les daturas sont des solanacées herbacées (Datura spp.) ou arborescentes (Brugmansia spp.). Six espèces ont été introduites en Nouvelle-Calédonie (3):D.cornigera Hook., D. ferox L., D. inoxia Mill., D. metel L., D. stramonium L., B. suaveolens (Humb. \& Bonpl. ex Willd.) Bercht. \& J.Presl. De par leur abondance naturelle, qui les rend facilement accessibles, deux espèces sont plus particulièrement consommées localement, $D$. inoxia et $B$. suaveolens. 
Datura inoxia (Figures 1,2) est une plante herbacée annuelle dressée, robuste et invasive, présentant des poils glandulaires. Ses feuilles matures sont grossièrement ovoïdes et découpées. La fleur blanche, dressée, possède un calice de 5 à $11 \mathrm{~cm}$ de long, 3 à 6 lobes de 13 à $20 \mathrm{~mm}$ de long, quelquefois incomplètement séparés. La corolle, de 12 à $19 \mathrm{~cm}$ de long, est blanche avec des veines vertes. Le limbe est ondulé et comporte une dizaine de lobes alternes, grossièrement triangulaires. Les étamines ne sont pas exsertes, les anthères ont 8 à $10 \mathrm{~mm}$ de long. Le pistil, de 10 à $14 \mathrm{~cm}$, est inséré loin derrière les anthères. Le fruit, qui est une capsule globuleuse et épineuse, d'où son nom de « pomme épineuse », contient jusqu'à 200 graines. $D$, inoxia, assez commune comme mauvaise herbe, n'est jamais cultivée et affectionne les terrains vagues ensoleillés et a été probablement importée d'Australie, le plus proche continent. Originaire du Mexique, d'Amérique du Sud et des Antilles, la plante a essaimé dans toutes les régions chaudes du globe.

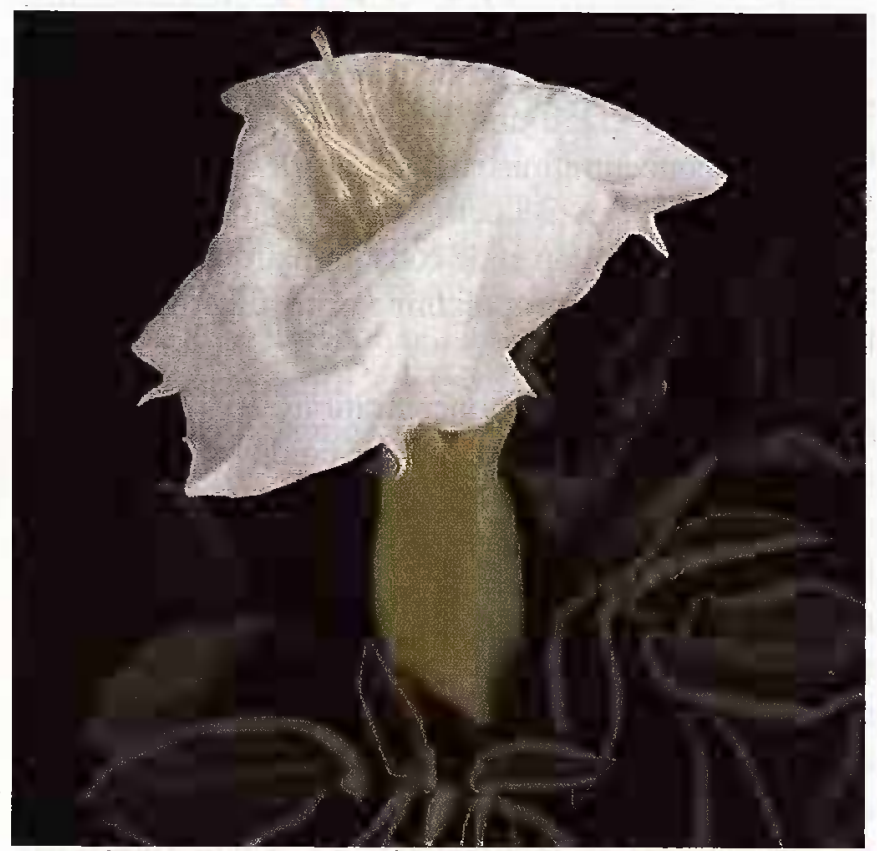

Figure 1 : Fleur de D. inoxia (orig. Internet).

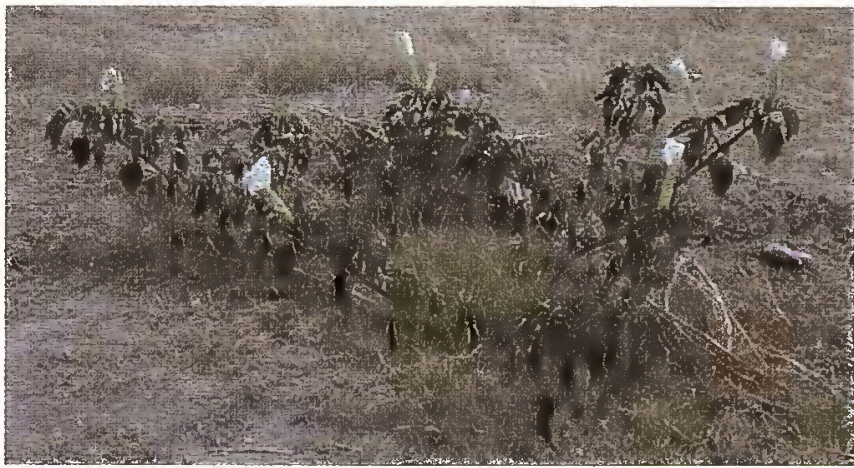

Figure $2: D$. inoxia (orig. Internet).
Brugmansia suaveolens (Figures 3, 4, 5) est une solanacée arborescente aux magnifiques fleurs pendantes en forme de trompette, au parfum agréable mais très entêtant, notamment le soir lorsque toutes les fleurs sont ouvertes. Les feuilles, ovoïdes et pileuses, ne sont pas découpées comme chez les daturas proprement dites. Comme $D$. inoxia, $B$. suaveolens est traditionnellement utilisée en Amérique du Sud pour ses propriétés hallucinogènes.

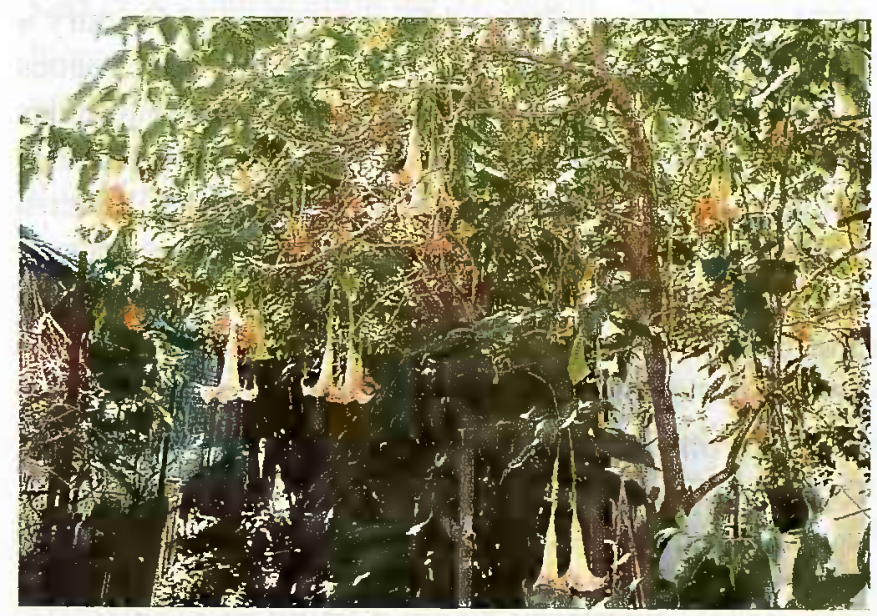

Figure 3 : B. suaveolens ou hybride (photo Y.B.).

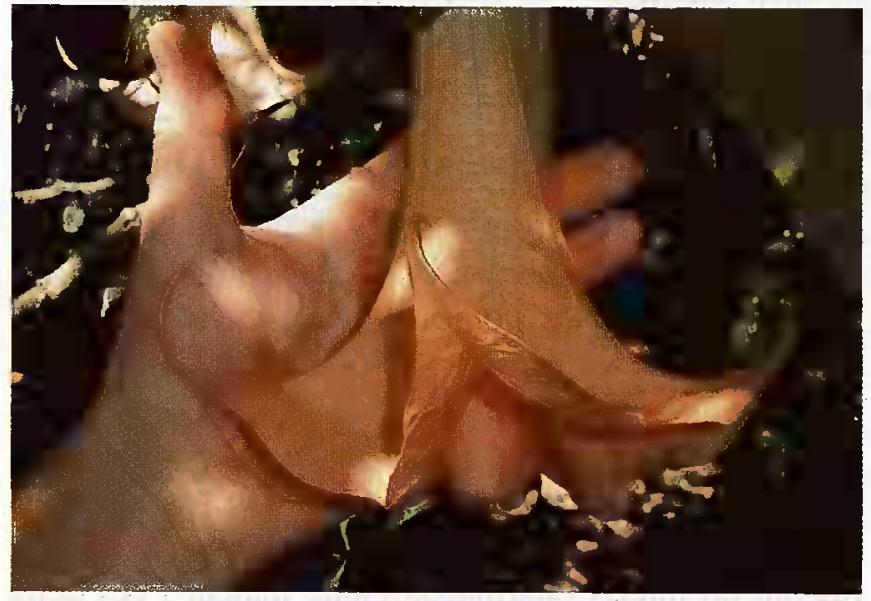

Figure 4 : Fleur de B. suaveolens ou d'hybride (photo Y.B.).

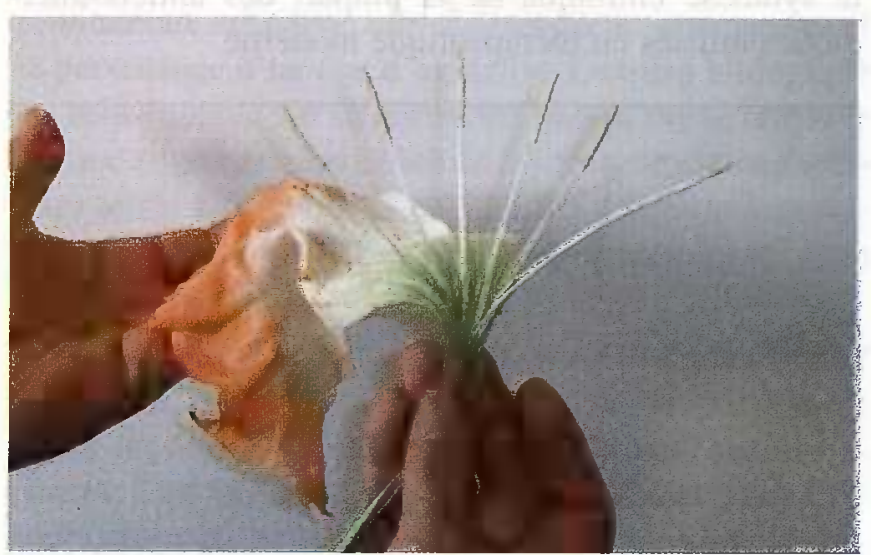

Figure 5 : Intérieur de cette même fleur (photo Y.B.). 


\section{Une toxicité connue depuis longtemps :}

La toxicité des daturas est connue depuis longtemps des populations amérindiennes d'Amérique du Sud. Deux médecins portugais : Garcia d'Orta (1501-1568) et Cristobald Acosta (vers 1525-1594) ont très bien décrit l'intoxication par le datura et les effets psychotropes engendrés, le traitement et l'usage par les prostituées de cette plante dans un but de soumettre chimiquement leurs clients afin de les détrousser par la suite. Ces récits ont été repris bien plus tard par F. Guerra en 1974 sous le titre «Sex and Drugs in the $16^{\text {th }}$ Century » (4). Dans ce texte où l'ampleur de l'usage des drogues est mis sur le compte d'un relâchement des habitudes sexuelles en Europe et en Amérique, mais aussi sur le compte du stress lié à la guerre du Vietnam, Guerra relève que l'usage des drogues psychotropes lors de relations sexuelles ou pour combattre la fatigue est un phénomène déjà clairement analysé par les médecins portugais et espagnols du $16^{\text {ème }}$ siècle.

L'utilisation des daturas ne fait pas partie des pharmacopées mélanésiennes, ces taxons étant d'introduction moderne dans le Pacifique. Traditionnellement, toutes les parties de la plante sont utilisées, afin de provoquer une sédation, faire disparaître la fatigue ou plus simplement dans un but de « défonce », état où prédomine un délire hallucinatoire. Les fleurs sont utilisées en décoction. Elles sont aussi fumées en association avec du cannabis.

\section{Les alcaloïdes tropaniques des Daturas :}

Les principes actifs responsables des effets pharmacologiques sont des alcaloïdes tropaniques. Ces alcaloïdes possèdent en commun un élément structural bicyclique azoté et sont des 8-méthyl-8-azabicyclo[3,2,1]octanes. L'atropine - mélange de $\mathrm{D}$ et L-hyoscyamine - et la scopolamine sont des esters d'alcools tropaniques (tropanol et scopanol, respectivement) (Figures 6 et 7). Ces deux substances actives des daturas ont un intérêt pharmacologique majeur : ces deux agents antimuscariniques ont été le point de départ de la synthèse chimique de la plupart des anticholinergiques utilisés en thérapeutique moderne.

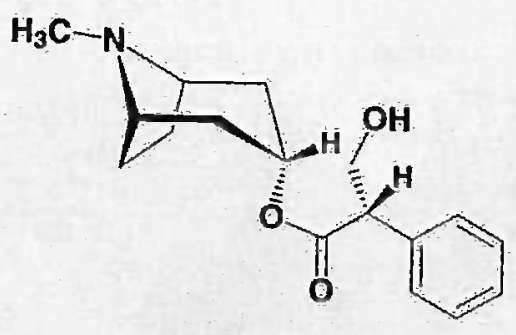

(-) -hyoscyamine

Figure 6 : Formule chimique de la hyoscyamine.

\section{Une nette prédominance de la scopola- mine :}

Anger et coll. ont déterminé les concentrations en atropine et en scopolamine du $D$. inoxia en NouvelleCalédonie (5). Différentes parties de la plante ont été analysées après extraction au dichlorométhane. La phase organique concentrée par évaporation a été reprise par le mélange acétonitrile-eau $(50 / 50)$ et analysée par CLHP-SM/SM. Dans la fleur, la scopolamine était présente à une concentration de $0,34 \mu \mathrm{g} / \mathrm{mg}$, le pistil $0,38 \mu \mathrm{g} / \mathrm{mg}$ et les graines $0,22 \mu \mathrm{g} / \mathrm{mg}$. Par rapport à la sopolamine, les auteurs ont noté que l'atropine était présente à des concentrations 100 fois moindres dans la fleur (5).

Nous avons analysé les fleurs et les feuilles de Brugmänsia suaveolens poussant à différents endroits de Nouméa, notamment celles d'une plante poussant devant l'entrée de l'Université utilisée éventuellement par des étudiants en quête de sensations fortes. Après séchage à l'étuve, les végétaux sont soumis à extraction au dichlorométhane. La phase organique concentrée est reprise par un mélange acétonitrile-eau (50/50) et analysée par CLHP/BD à l'aide d'une colonne Symmetry C8 ( $5 \mu \mathrm{m}, 4.6 \times 250 \mathrm{~mm}$. Waters) avec élution en gradient de concentrations d'acétonitrile/ tampon phosphate $\mathrm{pH}$ 3.6. Des décoctions sont réalisées selon les modes de préparation et de consommation locales et les concentrations en scopolamine sont déterminées par CLHP-BD.

Dans les feuilles, les concentrations en scopolamine varient de 0,80 à $10,60 \mu \mathrm{g} / \mathrm{mg}$ de poids sec, et dans les fleurs entières de 6,30 à $10,60 \mu \mathrm{g} / \mathrm{mg}$. Dans les tisanes, les concentrations en scopolamine vont de 30 à $37 \mathrm{mg} / \mathrm{l}$. Ces résultats sont en accord avec des études précédentes qui mesuraient de telles concentrations eñ scopolamine chez les Brugmansia. Ainsi $B$. sanguinea, reproduite par micropropagation in vitro de clones sélectionnés, est cultivée en Equateur en altitude pour la production de scopolamine. En 1990, la production équatorienne était estimée à 400 tonnes de feuilles sèches par an. D'après Bruneton, d'autres Brugmansia

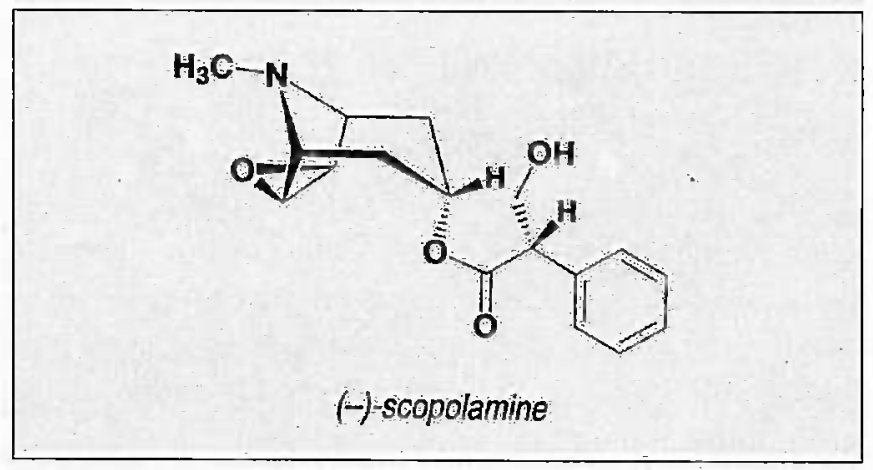

Figure 7 : Formule chimique de la scopolamine. 
et leurs hybrides pourraient être utilisés à des fins extractives (6). On notera cependant que dans notre région, en Australie, on préfère cultiver une autre solanacée : Duboisia myoporoides (aussi présente en NouvelleCalédonie) et $D$. leichhardtii, un genre qui peut contenir plus de 3\% de scopolamine dans les feuilles.

Les concentrations importantes de scopolamine des décoctions de fleurs de brugmansia expliquent que les usagers n'utiliseraient qu'une fleur pour réaliser la « Tisane du Diable » Iorsqu'ils utilisent $B$. suaveolens ou un hybride, alors qu'ils peuvent utiliser plusieurs fleurs lorsqu'il s'agit de Datura inoxia. Le délire hallucinatoire serait d'ailleurs rapidement atteint à l'aide de décoctions de brugmansia, tandis que la sédation pourrait prédominer avec $D$. inoxia.

Par CLHP/BD, nous n'avons retrouvé d'atropine ni dans les extraits végétaux ni dans les tisanes.

\section{Mécanisme d'action de la scopolamine au niveau central :}

La scopolamine est un antagoniste muscarinique à action centrale qui induit, chez les rongeurs, une hyperlocomotion. En effet, c'est un antagoniste muscarinique mixte bloquant aussi bien les récepteurs $\mathrm{M} 1$ que les récepteurs M2 du nucleus accumbens. Chez la femelle de rat Sprague-Dawley, l'antagonisme M1 entraîne une augmentation d'activité même en présence d'un blocage des récepteurs M2 responsable d'une libération d'acétylcholine, inhibitrice du mouvement (7). Dans une autre étude (8), après administration aigüe, la scopolamine induit une activation faible à modérée de type «extracellular signal-regulated kina$s e \gg(E R K)$ (kinase régulée par un signal extracellulaire) au niveau des noyaux gris centraux : système du striatum (putamen du noyau lenticulaire et noyau caudé), du noyau et de la coque du nucleus accumbens (un des noyaux du thalamus), du noyau latéral du lit de la stria terminalis, de l'amygdale centrale, des couches profondes du cortex préfrontal, de l'hypothalamus paraventriculaire. La scopolamine ne possède pas un fort potentiel addictif, l'activation de cet ensemble étant trop faible, même si une partie du système de récompense (comme le nucleus accumbens) est stimulé. Cependant, l'activation par la scopolamine des zones pré-citées peut expliquer les signes cliniques observés. Ainsi, l'activation du lobe temporal (amygdale) pourrait expliquer les hallucinations. Cliniquement, à faibles doses, la scopolamine est responsable de sédation ce qui peut en faire une drogue de soumission chimique, à plus fortes doses, le syndrome anticholinergique est majeur avec un tableau de délire atropinique marqué par des hallucinations. A ce stade, l'analyse toxicologique apporte une aide indiscutable au clinicien qui doit pouvoir écarter un épisode de psychose, particulièrement lorsqu'il s'agit d'un sujet amené aux Urgences de l'hôpital par la Police qui l'a trouvé errant seul dans la rue, après avoir commis des violences et tenant des propos incompréhensibles.

Nous rapportons les cas récents de 4 hommes âgés de 20 à 28 ans hospitalisés pour troubles du comportement après consommation de daturas.

\section{Cas cliniques :}

\section{Cas $\mathrm{N}^{\circ} \mathbf{1}$ : intoxication par D. inoxia.}

Nous décrivons ici le cas d'un. jeune patient de 20 ans admis aux urgences pour un état d'agitation avec hallucinations psychosensorielles survenant après la prise d'une infusion de fleurs de Datura inoxia.

Les antécédents de ce patient sont marqués par de multiples expériences toxicomaniaques avec consommation de cannabis et de champignons hallucinogènes en Métropole (amanite tue-mouche) ; on retrouve également une expérience au Datura stramonium dont les effets sont décrits comme proches de ceux du cannabis avec en plus la présence de palpitations. A ce propos, il est intéressant de noter que dans la péninsule malaise, $D$. fastuosa est utilisé pour ses propriétés «Ganja-like» (9). Les addictions de ce patient ont débuté avant son arrivée en Nouvelle-Calédonie et s'intègrent dans un contexte socio-familial difficile associant échec scolaire et rupture avec le milieu parental auquel s'ajoute un refus de prise en charge psychologique. Une prise de $D$. inoxia est retrouvée en juin 2003 : le patient aurait fumé une fleur séchée de datura mais dit n'avoir ressenti aucun effet.

Le 10 mai 2004, le patient est admis aux urgences pour un état d'agitation avec hallucinations psychosensorielles sans agressivité. Au moment de la prise en charge initiale, l'état hémodynamique montre une tachycardie régulière associée à une hypertension artérielle systolique supérieure à $180 \mathrm{mmHg}$.

A l'admission aux urgences, l'état hémodynamique s'est stabilisé ; l'examen olinique retrouve une mydriase bilatérale aréactive, une sécheresse des muqueuses et la persistance d'un état d'agitation. Le bilan biologique est remarquable par sa normalité puisqu' on ne retrouve qu'une augmentation isolée de la créatinine phosphokinase totale à $763 \mathrm{UI} / \mathrm{l}(\mathrm{N}<174 \mathrm{UJ} / \mathrm{l})$. Le traitement initial associe lavage gastrique et administration d'un sédatif par voie IV (clorazépate $50 \mathrm{mg}$ ).

Le patient est transféré en unité de soins intensifs pour surveillance. Le lendemain, on note la persistance d'une mydriase associée à des troubles de l'accommodation. Fait notable, la mydriase persistera environ 7 jours après la prise du toxique. L'évolution est rapidement favorable. 
Rétrospectivement, à l'interrogatoire, le patient reconnaît avoir préparé et ingéré une tisane préparée à partir de 6 fleurs de Datura inoxia séchées.

L'analyse toxicologique initiale dans le sang et les urines par CLHP/BD s'avère toutefois négative. La confirmation du diagnostic est apportée par l'analyse en LC-MS/MS d'un prélèvement capillaire effectué 3 semaines plus tard (4 juin 2004) (10). Les taux de scopolamine mis en évidence varient selon le segment de cheveux analysé et selon l'éloignement par rapport à la zone d'implantation. Nous obtenons ainsi 3 segments de $3 \mathrm{~cm}$ classés en fonction de la distance par rapport à la racine (de 0 à $3 \mathrm{~cm}$, de 3 à $6 \mathrm{~cm}$ et de 6 à $9 \mathrm{~cm}$ ) avec des taux de scopolamine de $14 \mathrm{pg} / \mathrm{mg}, 48 \mathrm{pg} / \mathrm{mg}$ et 43 $\mathrm{pg} / \mathrm{mg}$ respectivement. Ces taux de scopolamine et leur distribution tout au long des cheveux sont en faveur d'une prise chronique de la plante (10).

Le dosage d'atropine est resté négatif dans les prélèvements capillaires mais nous savons que les taux d'atropine contenus dans les fleurs de Datura inoxia sont jusqu'à 100 fois moindres que les taux de scopolamine (5).

Ce cas clinique montre l'intérêt des recherches de toxiques sur les matrices capillaires pour mettre en évidence une intoxication chronique par les alcaloïdes tropaniques.

\section{Cas $\mathrm{N}^{\circ} 2$ : intoxication par $B$. suaveolens.}

Patient de 20 ans admis aux urgences le 20/11/2004 pour suspicion d'intoxication au cannabis et au datura.

Le Service Médical d'URgence a été appelé par l'entourage du patient à la suite des troubles du comportement, avec notamment des difficultés à reconnaître les membres de sa famille, ainsi que des troubles circulatoires (irrégularité du pouls).

Dans les antécédents de ce jeune étudiant en lettres nous retrouvons des conduites addictives multiples plus ou moins chroniques avec consommation de cannabis, d'alcool, de tabac ; à noter également une réaction allergique au kava (Piper methysticum) le 08/04/2004 avec malaise vagal et manifestations oedémateuses ayant nécessité une hospitalisation et un traitement par corticoïdes avec oxygénothérapie.

Lorsque sa mère, infirmière, découvre son fils le matin du 20/11/2004, il présente une rougeur faciale et cervicale, il est incapable de la reconnaître et semble avoir un pouls irrégulier. Il reconnaît rapidement avoir fumé dans la nuit, entre $23 \mathrm{~h}$ et $6 \mathrm{~h}$ du matin, du cannabis et des fleurs séchées de brugmansia.

A l'admission aux urgences vers $10 \mathrm{~h}$ du matin, le patient présente un état de conscience normal sans désorientation ni agitation mais avec amnésie complète des faits. L'examen retrouve un syndrome anticholinergique : pupilles en semi-mydriase, réactives, séche- resse buccale et rougeur cervico-faciale. L'état hémodynamique est stable avec une légère tachycardie de repos à 90 battements par minute, sinusale ; l'électrocardiogramme est normal par ailleurs.

Les examens complémentaires sont normaux et une analyse toxicologique est demandée sur le sang et les urines.

L'analyse en LC/MS-MS retrouve dans le plasma un taux de scopolamine à $0,4 \mathrm{ng} / \mathrm{ml}$; aucune trace d'atropine n'est identifiée. Dans les urines, de l'atropine et de la scopolamine sont trouvées $(3,9 \mathrm{ng} / \mathrm{ml}$ et $8,5 \mathrm{ng} / \mathrm{ml}$, respectivement).

Le dosage urinaire des dérivés cannabinoïdes par méthode immunoenzymatique (AXSYM®; Abbott) est également positif.

Le traitement mis en place est une simple réhydratation parentérale associée à une surveillance. L'amélioration rapide de l'état du patient lui permet de quitter l'hôpital le soir même.

\section{Cas $N^{\circ} 3$ :}

Patient de 28 ans admis aux urgences pour une probable intoxication au datura.

Ce patient est amené le 01/02/2004 à 4 h 20 par des amis qui déclarent qu'il aurait bu la veille dans l'aprèsmidi de la «tisane à clochettes », préparée par un usager chronique qui en avait bu lui-même. Le patient aurait observé le préparateur de tisane marcher en titubant et en se cognant aux murs avant de tomber par terre et de s'endormir.

A l'examen clinique on note un syndrome atropinique avec délire hallucinatoire - le patient parlait avec des interlocuteurs absents - excitation psychomotrice, et obnubilation associés à une mydriase bilatérale, symétrique et réactive. L'état hémodynamique du patient est stable et le bilan para-clinique strictement normal.

Après administration de charbon activé, il est transféré en psychiatrie pour surveillance. L'évolution est simple avec récupération complète et rapide lui permettant de quitter l'hôpital le 03/02/2004.

Il est revu 15 jours plus tard pour prélèvement capillaire à visée toxicologique. L'analyse effectuée en LC-MS/MS reviendra négative ce qui montrerait les limites de ce dosage en cas de prise unique de Datura auquel il faudrait préférer un prélèvement immédiat de sang et d'urine.

\section{Cas $N^{\circ} 4$ :}

Patient de 22 ans admis aux urgences le 12/01/2005 à la suite d'une agression, dans un contexte de troubles du comportement d'apparition récente. Ce patient inconnu des services psychiatriques ne présentait aucun antécédent médical notable en dehors d'une consommation de cannabis et de datura en décoction (consommation supposée chronique). 
Cette hospitalisation apparaît en fait comme l'aboutissement d'une dégradation lente et inéluctable de l'état de santé de ce jeune homme ; en effet, les premières manifestations avaient débuté dans le courant du $2^{\text {ime }}$ semestre 2004 sous la forme de troubles psychiques $a$ minima se traduisant par des agressions physiques portant le plus souvent sur de jeunes femmes, et accompagnés d'un isolement avec tendance à la négligence et à la clochardisation progressive.

La décompensation se produit au début de l'année suivante sous la forme d'une nouvelle agression mais associée cette fois à des troubles psychiatriques majeurs (délire paranoïde, exaltation de l'humeur, discordance) posant la délicate question du diagnostic différentiel entre un délire sous l'influence d'un produit chimique et une bouffée délirante aigüe. L'état du patient nécessite son hospitalisation en secteur de psychiatrie avec placement d'office et instauration d'un traitement adapté.

L'analyse toxicologique par LC-MS/MS est menée sur un prélèvement de poils de barbe (son crâne étant rasé) compte tenu de la notion de prise chronique de datura. Les résultats étaient négatifs de même que la recherche de dérivés cannabinoïdes dans les urines.

Après 15 jours d'hospitalisation, l'état clinique du patient ne s'est guère amélioré ce qui semble parfaitement cohérent avec la négativité des recherches de toxiques, orientant fortement le diagnostic vers une pathologie psychiatrique, la consommation de datura étant probable (aux dires de l'entourage) mais occasionnelle. Seule l'évolution dans le temps et la récurrence éventuelle d'épisodes similaires permettra de faire la part entre un épisode délirant aigu et isolé et l'entrée du patient dans une psychose chronique (schizophrénie), la possibilité étant exclue d'appréhender jamais l'influence de la prise occasionnelle de datura dans l'apparition de ces troubles.

Ces cas cliniques prouvent l'utilité et la nécessité du recours à la LC-MS/MS dans la recherche et le dosage de la scopolamine et de l'atropine à partir du sang, de l'urine et des cheveux de sujets. En effet, les concentrations sont très faibles et la fenêtre de détection de ces alcaloïdes tropaniques dans le sang et l'urine trop étroite. Lors de prises uniques de datura, la recherche était négative dans les cheveux et dans les poils de barbe: cette négativité permet cependant d'éliminer une consommation chronique et de poser, pour le dernier cas, un diagnostic de pathologie psychiatrique.

La scopolamine est l'alcaloïde qui prédomine majoritairement dans les daturas herbacés et arborescents de Nouvelle-Calédonie. Ces cas cliniques montrent aussi la très nette prédilection des consommateurs envers les fleurs - partie de la plante la plus riche en scopolamine - les daturas fleurissant dans notre région plusieurs fois dans l'année.

\section{Le kava : rappel historique}

Le kava est une boisson rituelle du Pacifique préparée à partir des racines d'un poivrier : Piper methysticum. Forst. f. (Piperaceae) (Figure 8). L'espèce a été prédomestiquée en Papouasie Nôvelle-Guinée et/ou au Vanuatu à partir d'un ancêtre sauvage, Piper wichmannii C.DC. La plante fut ensuite diversifiée dans le Nord du Vanuatu au point que les deux tiers des cultivars connus y sont présents, sur les 120 environ que compte le Pacifique (Figure 9).

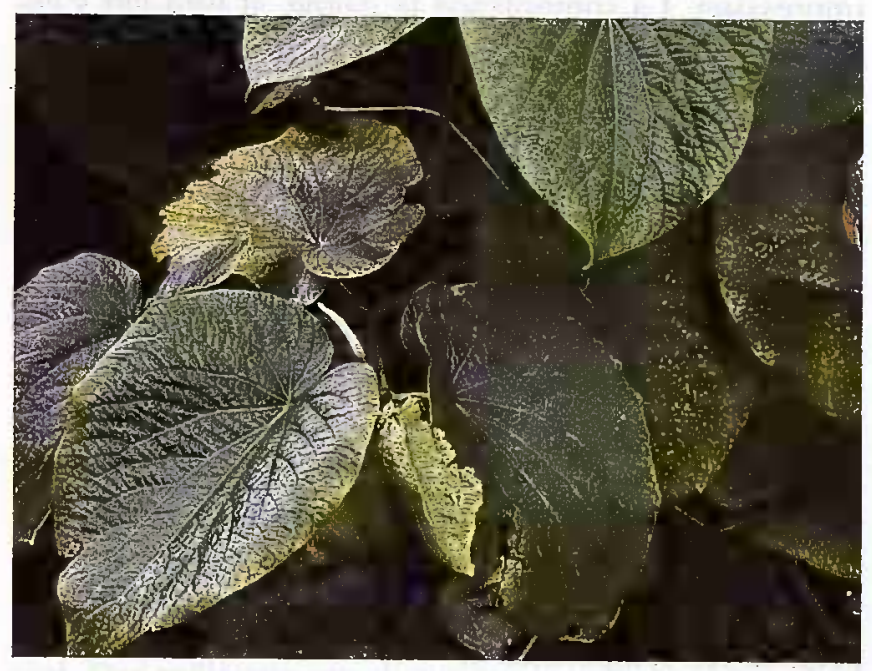

Figure 8 : Fleur femelle de kava (P. methysticum) entourée de feuilles cordiformes (photo P.C.).

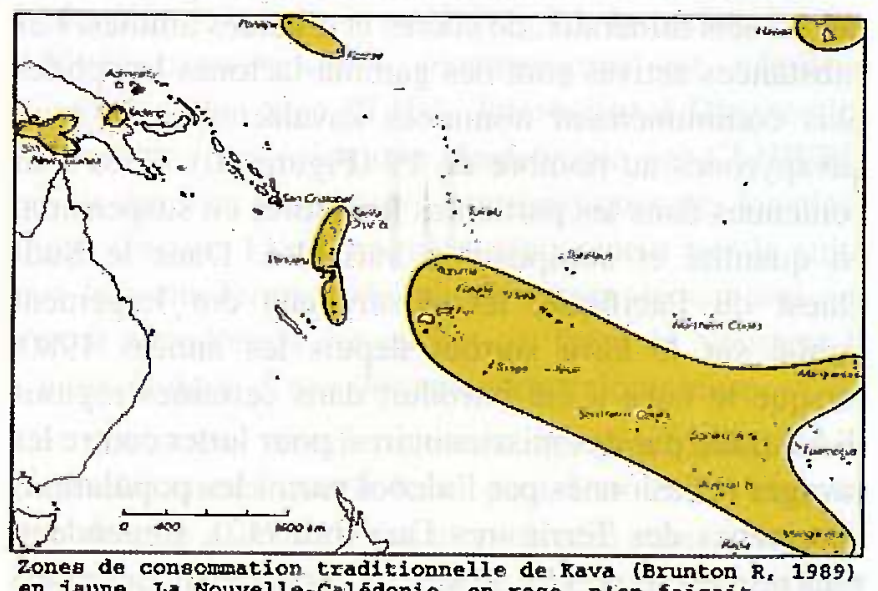

en jaure. La Nouvelle-caledonie, en rose, n'en faibait initialement pas paxtie.

Figure 9 : Répartition traditionnelle du kava d'après Brunton R., 1989. 
Dès 1886 le pharmacologue et toxicologue allemand Lewin étudie le kava, dont il décrit ainsi les effets (11): « lorsque les doses ne sont pas trop fortes, il s'établit, sans excitation physique ni psychique, un état d'insouciance heureuse, de bien-être et de contentement (...) Le kava adoucit les caractères. Jamais les buveurs ne deviennent coléreux, méchants, querelleurs ou bruyants, comme cela arrive avec l'alcool. Les indigènes et les blancs considèrent le kava comme moyen d'apaiser la douleur morale. On garde le contrôle de la conscience et de la raison. Mais lorsqu'on en absorbe des doses plus fortes, les membres deviennent las, les muscles semblent échapper aux ordres et au contrôle de la volonté, la démarche devient lente et mal assurée, les sujets ont l'air à moitié ivre. On sent le besoin de s'étendre (...) Le buveur succombe à l'épuisement et éprouve l'envie de dormir qui domine toute autre impression. La somnolence le gagne. Il finit par s'endormir (...) Le sommeil est analogue à celui de l'ivresse alcoolique et le sujet ne s'en laisse arracher que de mauvais gré. Lorsque les doses sont modérées, il s'établit de vingt à trente minutes après l'absorption du kava. Il dure environ deux heures, quelquefois même plus longtemps, et jusqu'à huit heures. (...) La duree dépend du degré de l'accoutumance du sujet. Lorsque le breuvage est concentré, c'est à dire lorsqu'il contient beaucoup d'éléments résineux, l'ivresse est beaucoup plus rapide. On trouve les buveurs couchés à l'endroit où ils ont bu. Avant de s'endormir, ils peuvent être pris d'un léger tremblement nerveux. (...) Pendant le sommeil, la sensibilité est diminuée. Aucune excitation ne précède les symptômes ».

\section{Les kavalactones}

La boisson est une émulsion contenant des résinoïdes, des fibres et de l'amidon dans une phase aqueuse chargée de sels minéraux, de sucres et d'acides aminés. Les substances actives sont des gamma-lactones lipophiles plus communément nommées kavalactones (KL) ou kavapyrones au nombre de 19 (Figure 10). Elles sont contenues dans les particules lipophiles en suspension, en quantité et composition variables. Dans le SudOuest du Pacifique, les Australiens ont largement publié sur le kava surtout depuis les années 1980 , lorsque le kava a été introduit dans certaines régions d'Australie par des missionnaires, pour lutter contre les ravages occasionnés par l'alcool parmi les populations aborigènes des Territoires Du Nord (12). Cependant, sans repère culturel lié au kava et recherchant des effets psychotropes importants, les Aborigènes de ces territoires avaient coutume d'en absorber des quantités très importantes, sans jamais se conformer à l'usage traditionnel océanien. Les autorités sanitaires décidèrent d'enquêter, ce qui stimula la réalisation de recherches scientifiques en Australie. De leur côté, les Allemands avaient très largement publié sur le sujet depuis le début du $20^{\text {ème }}$ siècle, la phytothérapie étant bien implantée dans leur culture médicale et le kava présent dans les répertoires pharmaceutiques depuis l'époque des «traders » allemands dans le Pacifique au $19^{\text {emo }}$ siècle.

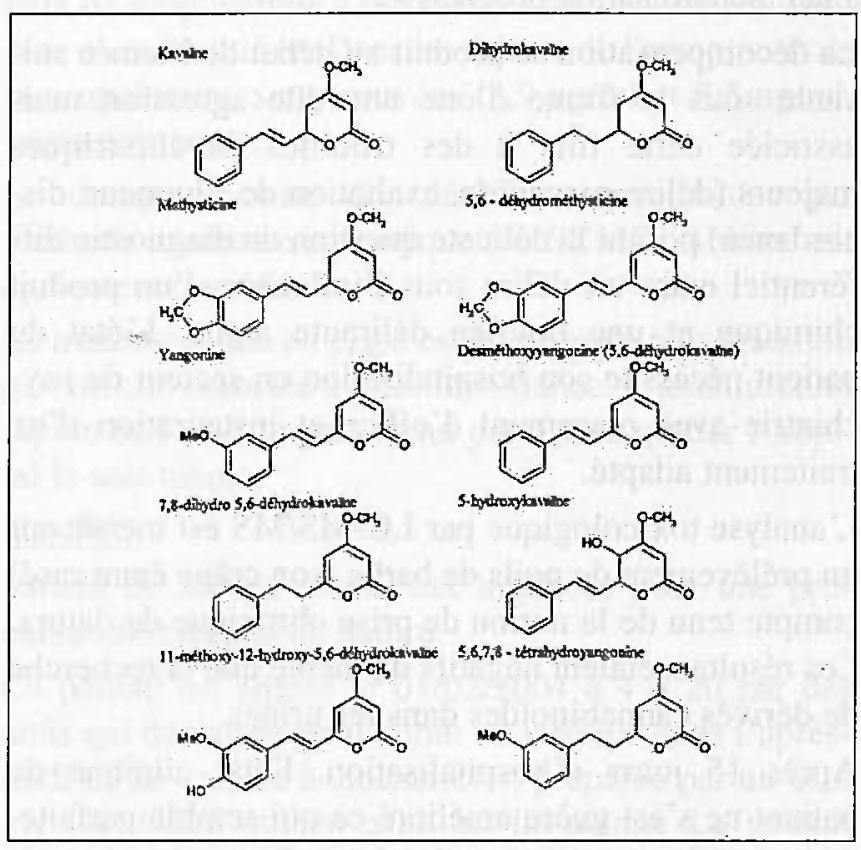

Figure 10 : Formules chimiques des principales kavalactones.

\section{Mécanismes d'action :}

Le kava est surtout réputé pour ses propriétés tranquillisantes, légèrement sédatives et myorelaxantes. Ces effets découlent de l'action centrale des kavalactones sur les récepteurs au GABA, au niveau de certains noyaux gris centraux (dont le nucleus accumbens) et peut-être aussi à l' activation des récepteurs corticaux (13). Le kava potentialise d'ailleurs les effets d'autres dépresseurs du SNC tels les benzodiazépines ou les barbituriques.

Des inhibitions multiples affectent les canaux cellulaires (sodium, potassium et calcium) et une activation touche les récepteurs au GABA ; il en résulte un effet stabilisateur de la membrane neuronale post-synaptique ainsi qu'un effet inhibiteur de l'augmentation des concentrations en calcium ionisé intracellulaire pré- et post-synaptique. Il en découle une mise au repos de la cellule nerveuse ainsi qu'une diminution de la transmission de l'influx nerveux. Les conséquences sont une sédation et une relaxation musculaire variant en fonction des susceptibilités des consommateurs. Ces effets apparaissent rapidement et sont relativement courts ; 
cependant; une absorption répétée ou massive entraîne un phénomène d'accumulation des substances actives et une prolongation de leur action cérébrale, dont l'importance est en relation avec la dose et la chronicité de la consommation. En cas d'associations, le kava renforce aussi l'action des autres dépresseurs du SNC de tous types, tranquillisants ou hypnotiques médicamenteux, alcool, cannabis.

L'alcool est utilisé volontairement pour, d'après les consommäteurs « caler le kava », c'est à dire ressentir à nouveau les effets du kava après avoir quitté le « bar à kava ». Le cannabis est consommé, en moyenne et en fonction des enquêtes, par la moitié des buveurs de kava. Le kava, seul, peut être consommé selon un mode abusif : le sujet parlera alors volontiers de dizaines de « sells de kava », de l'anglais shell ou coupes de kava, afin de décrire la quantité absorbée. Dans ce cas, il peut s'agir de personnes ayant connu auparavant une dépendance vis-à-vis de l'alcool.

\section{Cas médico-légaux :}

D'un point de vue médico-légal, de décembre 2002 à mai 2005 , nous avons répertorié treize cas où les kavalactones étaient retrouvées dans le sang d'auteurs ou de victimes d'accidents mortels (ayant entraîné dix décès et douze blessés graves), de suicides ou d'agressions (14). Ces chiffres sont cependant sous-évalués, les dépistages n'ayant pas été faits de manière systématique.

Par CLHB/BD, à l'aide d'une colonne Symmetry C8 (5 $\mu \mathrm{m}, 4.6 \times 250 \mathrm{~mm}$ Waters) avec élution en gradient de concentrations d'acétonitrile / tampon phosphate $\mathrm{pH}$ 3.6, les kavalactones sont détectées dans les prélèvements sanguins dans tous les cas à des concentrations variant de 180 à $5000 \mathrm{ng} / \mathrm{ml}$ pour la kavaïne, 680 à $12200 \mathrm{ng} / \mathrm{ml}$ pour la 7,8-dihydrokavaïne, 50 à 16500 $\mathrm{ng} / \mathrm{ml}$ pour le mélange méthysticine - 7,8-dihydrométhysticine. Les alcoolémies variaient de 0 à $2,47 \%$.

Nous rapportons ici quatre nouveaux cas médicolégaux. Trois sont survenus durant le seul mois de septembre 2005 ; ils concernent deux hommes impliqués dans deux rixes, dont l'une mortelle, et une femme retrouvée morte à son domicile, tandis que le dernier cas, survenu le $1^{\text {er }}$ janvier 2006, concerne une femme ayant porté plainte pour viol. Les âges sont compris entre 24 et 30 ans. Les analyses toxicologiques sont réalisées sur le sang par CLHP/BD selon le protocole précédemment décrit après extraction liquide / liquide à l'aide de Toxi Tubeß A (Varian).

Cas $\mathbf{N}^{\circ} \mathbf{1}$ : Homme de 28 ans travaillant dans un bar à kava et impliqué dans une rixe à son domicile. Sa châ̂ne stéréo étant réglée trop fort, son voisinage se plaint. Il s'en suit une altercation verbale qui dégénère ; sous les coups le sujet s'écroule puis décède. Un prélèvement sanguin intracardiaque est réalisé. L'alcoolémie (par CPG/FID) est négative, la recherche de dérivés cannabinoïdes par immunoenzymologie est positive (45 ng/ml) ; le screening toxicologique par CLHP/BD révèle des pics et spectres caractéristiques des kavalactones. Les deux agresseurs n'avaient qu'une alcoolémie de 0,40 et $0,61 \mathrm{~g} / \mathrm{L}$.

Cas $\mathbf{N}^{\circ} \mathbf{2}$ : Homme de 20 ans, se dispute avec sa femme et reçoit un coup de couteau de-cuisine dans la poitrine. Admis en urgence à l'hôpital, il est sauvé. Un prélèvement de sang veineux est effectué. L'alcoolémie est négative, la recherche de dérivés cannabinoïdes par immunoenzymologie est positive $(45 \mathrm{ng} / \mathrm{ml})$; le screening toxicologique par CLHP/BD révèle des pics et spectres caractéristiques des kavalactones.

Cás $N^{\circ} 3$ : Femme de 24 ans trouvée au matin morte dans son lit. Un prélèvement sanguin intracardiaque est effectué. L'alcoolémie est négative, il n'y a pas de trace de stupéfiants; le screening toxicologique par $\mathrm{CLHP/BD}$ révèle des pics et spectres caractéristiques des kavalactones et des traces de cyamémazine. L'histoire clinique révèle que cette personne souffrait d'encéphalopathie entraînant des crises comitiales ; le sujet souffrait aussi de psychose (schizophrénie). Il était traité par acide valproïque, clonazépam, qui n'ont pas été retrouvés au screening. La cyamémazine a été rajoutée au traitement à $17 \mathrm{H00}$ le soir du décès et l'hypothèse d'une mort subite par syndrome du QT long est retenue.

Cas $\mathbf{N}^{\circ} 4$ : Femme de 30 ans ayant porté plainte pour viol. Des prélèvements divers sur la victime (pour sérologies, test de grossesse, recherche de toxiques sanguins, écouvillonnages...) sont effectués aux Urgences de l'Hôpital sur réquisition de la Police Nationale. L'alcoolémie est positive $(1,31 \mathrm{~g} / \mathrm{L})$, la recherche des dérivés cannabinoïdes plasmatiques est négative (Cannabis One-Step ELISA, International Diagnostic) le screening toxicologique plasmatique par CLHP/BD révèle des pics et spectres caractéristiques des kavalactones (Figure 11). L'enquête déterminera par la suite que la jeune femme désirait se venger de son ami qui l'avait abandonnée sur un îlot, au large de Nouméa. Il s'agissait donc d'une dénonciation calomnieuse.

Ces quatre cas récents, ajoutés aux précédents, montrent clairement que le kava peut être utilisé comme une drogue d'abus. Il est alors ingéré massivement à des doses n'ayant plus aucun lien avec la tradition océanienne ; le kava est aussi fréquemment utilisé en association à d'autres psychotropes (cannabis, alcool ou médicaments). Le poly-usage l'associant à l'alcool et au cannabis, à but avoué de « défonce », est communément nommé « trithérapie » ou « triathlon » par cer- 
tains usagers eux-mêmes (15).

La consommation de kava était historiquement inconnue en Nouvelle-Calédonie, les « bars à kava » y fonctionnent sur un mode commercial qui s'éloigne du modèle traditionnel importé du Vanuatu, et cette boisson tend donc à devenir un produit de consommation courante ; mais à la différence de l'alcool, des médicaments et des drogues, c'est aujourd'hui le seul psychotrope qui ne soit pas réglementé - au contraire du cas australien - en Nouvelle-Calédonie ainsi que dans de nombreux autres pays du Pacifique.

\section{Conclusion}

Il a été montré que les substances addictives partageaient la propriété d'induire une forte activation de type ERK dans un nombre limité de structures incluant le noyau du nucleus accumbens, plusieurs composants de l'amygdale (coque du nucleus accumbens, l'amygdale centrale, noyau latéral du lit de la stria terminalis), ainsi que les couches profondes du cortex préfrontal (8). Cette activation étant médiée par des récepteurs dopaminergiques D1. Ni la scopolamine ni les kavalactones à elles-seules n'activeraient cet ensemble dans sa totalité ni de façon suffisante, leur pouvoir addictif est donc relativement faible, cela même si certaines structures du cerveau comprises dans le « système de récompense », comme le nucleus accumbens, sont stimulées (Figure 12).

Cependant, ces psychotropes permettent de renforcer très efficacement les effets de l'alcool et du cannabis dans un but de recherche de sensations très fortes, de « défonce »; ce qui amène l'expérimentateur à adopter des conduites à risque pouvant finalement le conduire à l'hôpital ou bien sur la table du légiste.

Du rite social ou culturel aux abus : sans références traditionnelles, l'usage des plantes psychotropes est souvent dévoyé et peut ouvrir la porte aux abus. Il s'agit surtout d'un poly-usage associant plantes, alcool et cannabis à but avoué de « défonce » par potentialisation des effets de ces consommations. Le développement du kava est parfois présenté comme un retour à la tradition dans divers pays du Pacifique ou comme le développement d'une "néo-tradition » en Nouvelle-Calédonie, mais l'ensemble de ce phénomène est la cause de certains abus, dans une société par ailleurs relativement permissive. Ces abus doivent être officiellement exposés et dénoncés par des instances gouvernementales. Le « secret-défonce » ainsi levé devrait permettre de définir une réglementation plus adaptée, notamment concernant les poly-consommateurs.

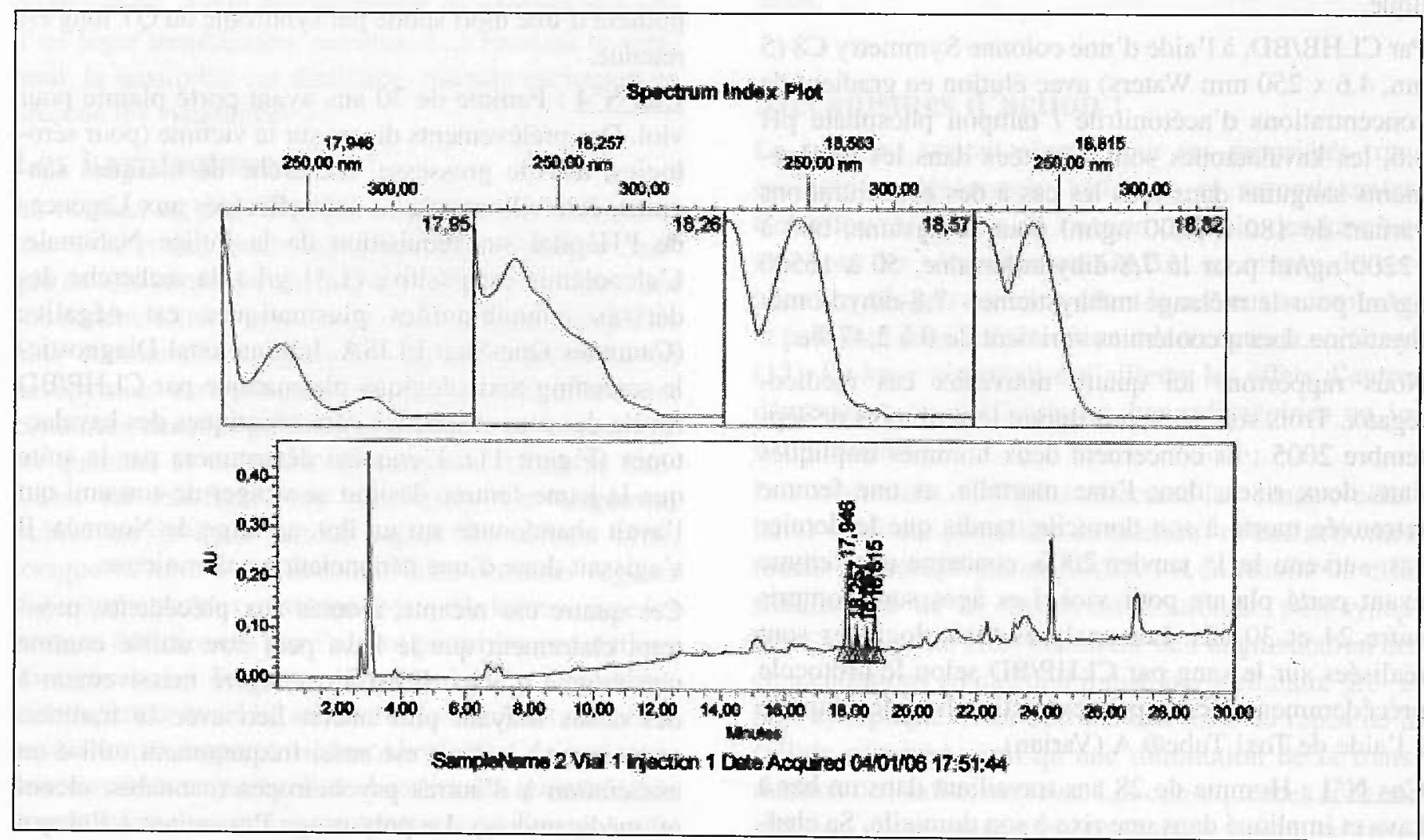

Figure 11 : Chromatogramme et spectres de kavalactones et métabolites, cas $N^{\circ} 4$ (spectres UV de gauche à droite : dihydrométhysticine, méthysticine, kavä̈ne, p-Irydroxykavä̈ne). 


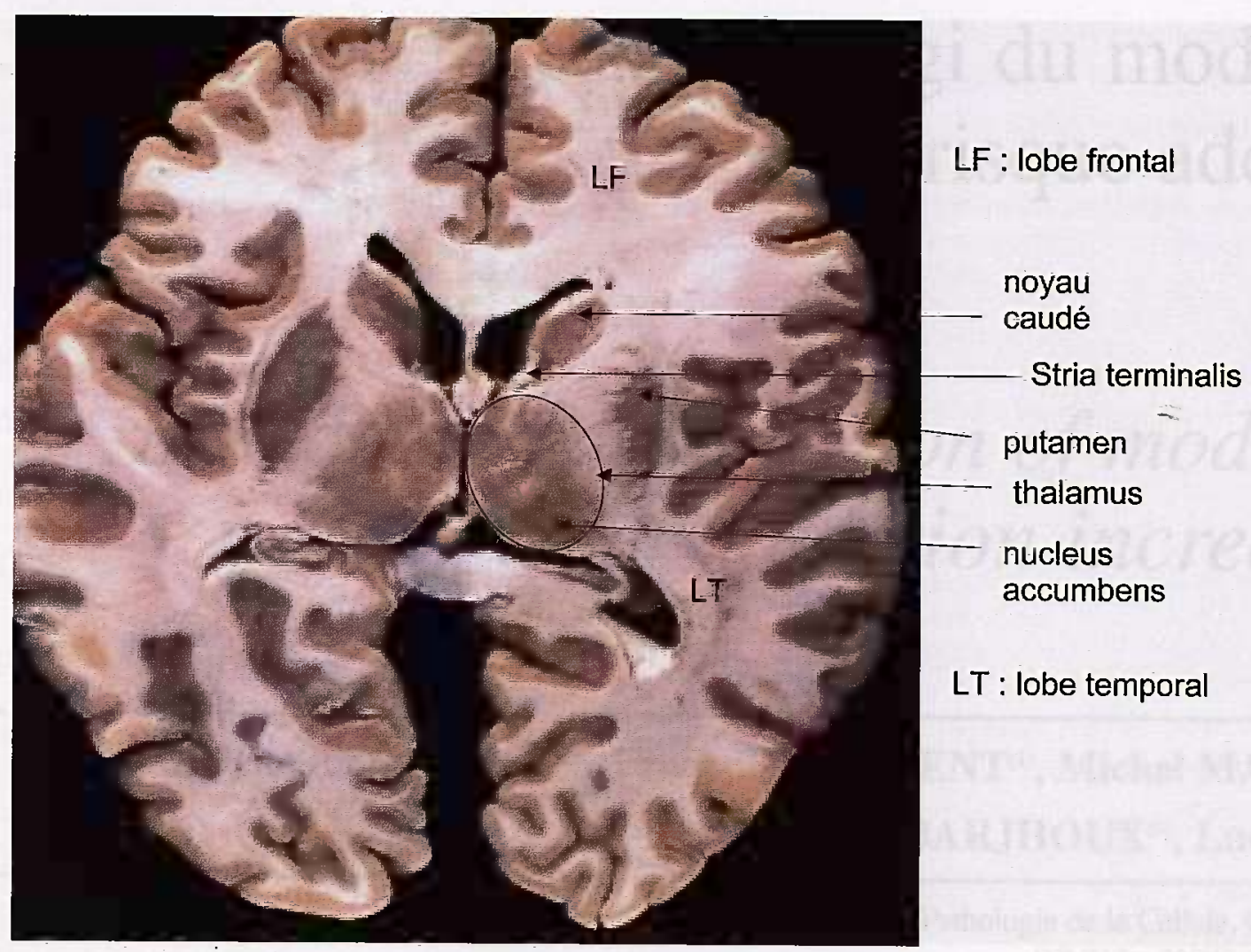

Figure 12 : Coupe horizontale du cerveau humain à travers le milieu du thalamus, montrant les principales zones stimulées par les substances addictives (d'après Willians èt coll.).

\section{Références}

1. Barguil Y., Cirimele V., Charlot J.Y., Duhet D, Villain M., Kintz P., Choblet E. Potency of cannabis seized in New Caledonia (2004 - 2005). Séoul : Proceedings of the TIAFT 43rd meeting, $2005: 152$.

2. Shulgin, A.T. The narcotic pepper: the chemistry and pharmacology of Piper methysticum and related species. Bull. Narcotics, 1973 ; 25 : 59-74.

3. MacKee H.S. S. Catalogue des plantes introduites et cultivées en Nouvelle-Calédonie. Paris : Museum National d'Histoire Naturelle, $1994: 130-1$.

4. Guerra F. Sex and drugs in the 16th Century. Br. J. Addict. $1974 ; 69: 269-90$.

5. Anger J.P., Villain M., Baert A., Kintz P. Le datura : une plante oubliée de la pharmacopée mais qui semble de plus en plus plébiscitée par les jeunes. Annal. Toxicol. Anal. $2004 ; 16(3): 166$.

6. Bruneton J. Alcaloïdes tropaniques. In : Pharmacognosie. Phytochimie. Plantes médicinales. Paris : Tec \& Doc Lavoisier, 1999 ; 805-24.

7. Chau D.T., Rada P., Kosloff R.A., Taylor J.L., Hoebel B.G. Nuccleus accumbens muscarinic receptors in the control of behavioral depression: antidepressant-like effects of local M1 antagonist in the Porsolt swim test. Neurosci. $2001 ; 104$ (3): 791-8.

8. Valjent E., Pagès C., Hervé D., Girault J.A., Caboche J. Addictive and non-addictive drugs induce distinct and specific patterns of ERK activation in mouse brain. Eur. J. Neurosci. $2004 ; 19: 1826-36$.
9. Quek K.C., Cheah J.S. Poisoning due to ingestion of the seeds of kechubong (Datura fastuosa) for its ganja-like effect in Singapore. J. Trop. Med. Hyg. 1974 : 77 (5) : 111-2.

10. Kintz P., Villain M., Barguil Y., Charlot J.Y., Cirimele V. Testing for atropine and scopolamine in hair by LCMS/MS after Datura inoxia abuse. J. Anal. Toxicol. 2006, soumis pour publication.

11.Lewin L. Le Kawa-kawa. In Phantastica. Paris : Petite Bibliothèque Payot, réed. $1970 ; 230-9$.

12. Cawte J. Parameters of kava used as a challenge to alcohol. Austr. N. Z. J. Psych. 1986 ; 20 : 70-6.

13. Boonen G.,. Pramanik A., Rigler R., Häberlein H. Evidence for specific interaction between kavain and human cortical neurons monitored by fluorescence correlation spectroscopy. Planta Medica. $2000 ; 66$ : 7-10.

14. Barguil Y.; Tarbah F., Shaheen A., Duhet D., Choblet E., Daldrup Th. Thirteen forensic cases related to kava consumption in New Caledonia (2002 - 2005). Séoul : Proceedings of the 43rd TIAFT meeting, 2005: 83 .

15. Laroche S., Cabalion P., Barguil Y. Typologie de la consommation de kava en Nouvelle-Calédonie, profils d'après enquêtes " à dires de buveurs ». Ethnopharmacologia. $2005 ; 35: 19-31$.

16. Brunton R. The abandoned narcotic, kava and cultural instability in Melanesia. 1989; Cambrige studies in social antropology. Cambrige University Press. 219 pp.

17. Williams T.H., Gluhbegovic N., Jew J.Y. Virtual Hospital. www.vh.org, site consulté en décembre 2005. 